Voix et Images

\title{
V.7 BJ/NBJ : un nouvel imaginaire
}

\section{Claude Beausoleil}

Volume 10, numéro 2, hiver 1985

La barre du jour / La nouvelle barre du jour

URI : https://id.erudit.org/iderudit/013882ar

DOI : https://doi.org/10.7202/013882ar

Aller au sommaire du numéro

\section{Éditeur(s)}

Université du Québec à Montréal

ISSN

0318-9201 (imprimé)

1705-933X (numérique)

Découvrir la revue

Citer cet article

Beausoleil, C. (1985). V.7 BJ/NBJ : un nouvel imaginaire. Voix et Images, 10(2),

167-171. https://doi.org/10.7202/013882ar d'utilisation que vous pouvez consulter en ligne.

https://apropos.erudit.org/fr/usagers/politique-dutilisation/ 


\title{
V.7 BJ/NBJ: un nouvel imaginaire
}

\author{
par Claude Beausoleil, Collège Edouard-Montpetit
}

L'Homme ruine inlassablement le monde, et la recherche formelle, elle aussi, ruine la convention du monde. En ce sens, elle est révolutionnaire. C'est donc dire qu'une revue littéraire n'a rien à perdre avec l'avant-garde et la recherche.

Le Quartier latin, 16 février 1967, p. 4-5.

il fouille ses avenues le temps qu'il fait/dans tes chevelures d'aujourd'hui

La Barre du jour, Michel Beaulieu, mars-avril 1977, no 55, p. 53.

Les phrases se posent les unes à la suite des autres et chacune fabrique la totalité du projet en montrant les autres phrases et en étant montrées par elles.

La Nouvelle Barre du jour, Nicole Brossard, novembre 1982 , no $118-119$, p. 180.

à ceux qui écrivent, cherchent

.La BJ et la NBJ ont-elles proposé un nouvel imaginaire? Il s'agit de définir des enjeux repérables dans le corpus en suivant des pistes qui disent par leur aptitude aux rassemblements thématiques ou parfois au langage mimétique, ce qui s'est faufilé dans les travaux d'écriture publiés dans le cadre de cette revue depuis sa fondation en 1965. Question parfois de territoire, question aussi de pratiques vérifiables dans les nombreux textes qui participent de cette chose toujours un peu anthologique qui constitue une revue. Par des mots devenant point de vue je circulerai dans ces réseaux tissés eux-mềmes de mots, de langage. Cette position d'analyse se risque du côté du fragment. Cette attitude, j'y reviendrai, me semble rendre compte dans une certaine mesure d'une spécificité pratique amenée dans ces écritures. Définir le projet de cette revue en un mot serait impossible. C'est dans la juxtaposition palimpseste de particules que l'on peut déchiffrer le territoire mais

«Rien n'est plus fragile qu'un territoire. Tôt soumis à l'ouverture. Dès sa formation, et se formant contre elle. L'inverse de l'arène qui par ce qui s'y joue se tient close sur elle-mème par l'intérieur, par le jeu tragique qui s'y joue. 
Espace aux bords nets comme le fil du rasoir. Espace décisif, espace décisoire.)

Daniel Klébaner, Poétique de la dérive, «Le Chemin» Gallimard, 1978, p. 131.

\section{les mots choisis}

Les mots choisis sont: l'imaginaire, le fragment, l'extrait, le texte, la citation et le corps. À partir d'eux, le puzzle se recompose et donne à voir, montre, la surface et le tracé d'une lecture possible de cette revue dont les avenues sont multiples. Ce fonctionnement autour de certains mots veut permettre une approche même friable du comment cette revue aurait proposé un nouvel imaginaire.

\section{l'imaginaire}

C'est a l'ensemble des signes formant un tableau aux contours délimités que se réfère le mot imaginaire. C'est à partir du réel de ce qui est véhiculé dans l'écriture que l'on peut déduire les préoccupations d'un imaginaire. Sur ce plan la BJ/NBJ a peu glosé quant aux perspectives d'orientation (cf. les notes liminaires souvent brèves qui viennent jalonner les politiques éditoriales). L'imaginaire de cette revue au second titre enchâssé dans le premier pourrait peut-être se jouer dans l'ajout de cet adjectif «nouvelle» qui est là pour inscrire et faire lire la volonté d'aller explorer plus avant le textuel. $\mathrm{La}$ BJ ne-changera pas vraiment de nom en septembre 1977 (no 58). La N (et ici je souligne) BJ ne vient que préciser ce désir ponctuel du neuf. Devant cette hypothèse, on voit que depuis la BJ ce qui pointe à l'horizon - les chamberdements et les questions de modes et de courants sont là à tenter toujours, à reformuler, à réinscrire que le travail d'écriture est une activité axée sur le nouveau. L'imaginaire prôné dans la BJ/NBJ pourrait bien être cette adhésion au nouveau, aux remises en question, au poreux des formes qui changent. Je crois dans une certaine mesure que de façon tautologique on pourrait écrire que le nouvel imaginaire de la BJ/NBJ est le nouveau. C'est dans ce territoire toujours mobile puisque par définition le nouveau contient une nécessité de déplacement que les textes posent, misant leurs contours, prenant dans l'aventure du langage les stimulations menant à cette impulsion du renouvellement.

\section{le fragment}

L'imaginaire en écriture passe par la forme. C'est dans ce concret que l'on peut déceler sa spécificité, son lieu. Dans les travaux publiés sous l'étiquette de la BJ/NBJ, le fragment m'apparaît être une condition matérielle fréquente du texte comme gènre. C'est stratégiquement par le fragment que ces écritures se sont souvent distanciées ou opposées à d'autres démarches d'écriture plus conventionnelles défendues dans d'autres revues. Visuellement et nommément, le fragment montre sa 
présence comme mode d'emploi possible de ce qui sera nommé tardivement «la nouvelle écriture». Il y a dans cette notion de fragment, implicitement, la notion d'ouverture, comme si entre ces morceaux d'écritures se déplaçaient d'autres variantes. . En présentation au numéro anthologique Poésie 80 (NBJ 92-93) Michel Beaulieu écrit: «Elle (l'anthologie) permet, grâce à la brièveté relative du temps qu'elle recouvre, de saisir en quelque sorte d'un coup d'oeil et de confronter, car tel est le mérite fondamental de cette entreprise, des fragments d'ouvres qui se construisent simultanément au moment même où elle parâtt» (p. 8). Si on retient comme hypothèse que la revue est une sorte d'anthologie mobile, trouee, il y a dans le fragment lu comme moteur quelque chose de prismatique permettant un complément, une interaction entre des facettes d'écritures singulières.

\section{l'extrait}

Il y a beaucoup de textes qui se donnent comme extrait montrant par là qu'ils sont en chantier. Il pourrait s'agir ici de la fonction laboratoire du travail de l'écriture en transformation. L'extrait est comme un prélèvement organique qui donne un aperçu d'une ouvre complète. On retrouve ce mécanisme ramifié dans le «à paraître». Voir les travaux d'auteurs comme André Roy, François Charron ou Jean Yves Collette (nos 74, 86, 88, 92, 93, etc.) comme exemples d'échantillonnages progressifs de l'oeuvre en élaboration. Un auteur comme. André Roy a beaucoup publié en extrait ce qui allait devenir des titres aux Herbes rouges après certains remaniements. L'extrait et l'à paraitre viennent, il me semble, renforcer l'idée de définir ou de lire une revue comme étant une anthologie où se dispersent les pistes.

\section{le texte}

Le mot texte est important pour situer la portée conceptuelle d'une revue comme la BJ/NBJ. C'est en effet par ce mot que l'on peut saisir l'ambigu mais constant questionnement des genres littéraires traditionnels. On peut remarquer cet effet du texte surtout dans la NBJ mais on retrouve l'origine de l'utilisation du terme dans la BJ. Le mot texte est apparu pour la première fois dans le sommaire du numéro 3-5, en 1965. Ici le terme est utilisé comme information et non comme catégorie. Mais tout de même on peut noter qu'à cette époque il était plus habituel de parler de poème, etc. Le mot texte, d'ailleurs partout composé en capitales, apparaît comme cristallisateuí de pratiques d'écriture dans le numéro 14 (été 1968, pp. 63-70), dans l'article de Claude Bertrand: "Introduction à l'histoire de la rupture"; ainsi que dans le numéro 15 (août-septembre 1968) consacré à Jean-Michel Valiquette, «lè plus étrange écrivain du Québec», sous la signature de Claude Bertrand («Chevauchement»; p. 7-9). C'est le poète Michel Beaulieu qui le premier dans la BJ (no 17-20, janvier-aout 1969) consacré aux Automatistes, utilisera comme titre d'un témoignage le mot texte (p. 271). Au fil des numéros et des années, ce mot finira par presque signifier un nouveau genre littéraire, genre littéraire qui contiendrait en sa définition des travaux d'écriture qui implicitement ou explicitement ne 
veulent pas se ranger sous les mots poème ou prose. Le mot texte serait une façon d'accéder à la modernité comme état d'esprit, comme pratique également. Le numéro 103, intitulé l'Infratexte, montrait en quelque sorte une exploration théorique de la question appliquée à des lectures.

\section{la citation}

On remarque dans la BJ/NBJ une grande utilisation de la citation. Cette mixture du texte original et d'intertextes se présentant sous la forme de la citation vient souligner l'aspect en ébullition de ces écritures qui s'écrivent souvent dans (ou à partir) d'autres écritures théoriques ou fictionnelles. À ce titre les travaux de Yolande Villemaire, dans des textes "Pour une parthéongenèse de la parole «hystérique»" (no 50, Femme et Langage, p. 37, hiver 1975) sont exemplaires de cette utilisation de la citation qui vient relancer, accompagner le texte original comme pour le faire parler au pluriel, avec échos. La citation dans le texte moderne joue un rôle de bricolage qui découle de l'aspect expérimental des démarches d'écriture. Citer, c'est afficher le polyphonique, la brèche par où, et dans laquelle, on parle autrement et avec d'autres.

le corps

On ne peut parler de la BJ/NBJ sans rappeler le type d'osmose qui s'y est proposée entre la textualité et la sexualité, entre le corps et les mots (cf. des numéros comme Le corps les mots l'imaginaire, no 56-57, mai-août 1977, ou encore Ce qui les séduit no 127-128, juin 1983). Le corps, dans cette revue, est le pivot de plusieurs recherches. On l'utilise comme tremplin, comme source de ce qui donnera l'écrit. Si cette analogie corps/texte a été parfois décriée, il n'en demeure pas moins qu'elle a permis un travail d'élucidation au sujet des mécanismes de l'écriture. Toute écriture est aussi un acte physique, un travail. En liant intimement les mots et le corps on $\mathrm{a}$, je crois, éclairé une lecture matérielle des enjeux de l'écriture moderne. Plusieurs auteur(e)s, comme Nicole Brossard, André Roy, Denise La Frenière, Hugues Corriveau ou Denise Desautels, ont donné à cette analogie une multiplicité de significations. Ce passage de l'écriture par le corps et ses épreuves (sexualité, psychanalyse, quotidien, forme, plaisir) a donné des versions éclatées de cette binarité opératoire qu'il ne faudrait pas rejeter trop rapidement du simple fait qu'elle soit devenue, et là justement par le travail des textes, plus évidente. Je crois que ce rapprochement corps/texte a produit des résultats très intéressants dont la lecture nous laisse mesurer un des supports de l'imaginaire tel qu'il est traversé dans la BJ/NBJ.

les mots dérivent

Si nous nous représentons les faits autrement qu'ils ne sont, certains jeux de langage perdent de l'importance, d'autres en gagnent. Et c'est 
ainsi, peu à peu, que se modifie l'emploi du vocabulaire du langage.

Ludwig Wittgenstein, De la certitude, «Idées», Gallimard, 1976, p. 43.

Dès son premier éditorial, la BJ se pose dans le champ du travail littéraire tout en ne niant pas ce qui se passe dans le social. Cette démarche sera toujours présente à travers les livraisons ultérieures. La littérature sera toujours centrale, mais les courants idéologiques viendront sous forme référentielle ou thématique transformer les indices des recherches proprement textuelles. C'est dans cette conjoncture que l'on peut lire le travail de l'imaginaire, un imaginaire qui n'est pas désincarné malgré son allégeance à l'exploration formelle. En témoignent des numéros comme l'Image parle (no 35-37, automne 1972), influencé par les mouvements de la contre-culture, ou encore Onze analyses (no 39-41, printemps-été 1973) sous l'influence des courants européens concevant l'analyse littéraire comme science et, de façon certaine, les nombreux numéros de femmes travaillant dans l'impact des réflexions et des démarches féministes. Donc il ne faudrait pas lire l'imaginaire de la BJ/NBJ comme un système évoluant en vase clos. Bien au contraire, cette revue a souvent pointé la transformation sociale et idéologique. Et si on oublie un peu cet aspect d'insertion dans les préoccupations sociales et idéologiques, c'est que la fiction emprunte parfois des voies complexes, elliptiques. Tout changement, même textuel, est tributaire d'un contexte et si, dans la BJ/NBJ, les mots dérivent dans la page, cette page, c'est toujours aussi le réel. 